\title{
Increased expression of phosphatidylinositol 3-kinase p110 $\alpha$ and gene amplification of PIK3CA in nasopharyngeal carcinoma
}

\begin{abstract}
Molecular alterations in PIK3CA oncogene that encodes the p110 catalytic subunit of phosphatidylinositol 3-kinase (PI3K p110Ŭ) are commonly found in human cancers. In this study, we examined the expression of PI3K p110Ŭ and PIK3CA gene amplification in 74 nasopharyngeal carcinoma (NPC) cases. Immunohistochemical staining demonstrated overexpression of PI3K p110Ŭ protein in $44.6 \%$ (33/74) of NPCs and $4.8 \%(2 / 42)$ of the adjacent normal nasopharyngeal mucosa. Copy number of PIK3CA gene was successfully analyzed in 51 of the total NPC cases and 19 non-malignant nasopharynx tissues by quantitative real-time PCR. Using mean +2 (standard deviation) of copy numbers in the nonmalignant nasopharynx tissues as a cutoff value, PIK3CA copy number gain was found in 10 of $51(19.6 \%)$ NPC cases. High PI3K p110Ŭ expression level was correlated with increased PIK3CA copy number (Spearmanô rho $=0.324, \mathrm{P}=0.02$ ). PI3K p110Ŭ expression and PIK3CA copy number did not associate with Akt phosphorylation, and patient and tumor variables. This study suggests that PI3K p110Ŭoverexpression, which is attributed, at least in part, to PIK3CA gene amplification, may contribute to NPC pathogenesis. However, these molecular aberrations may not be responsible for activation of Akt signaling in NPC.
\end{abstract}

Keyword: Akt; Immunohistochemistry; Nasopharyngeal carcinoma; PIK3CA 